\title{
Quality of Life and Functional Independence of Hip Fracture Patients: Data from a Single Center Follow-Up Study in Sri Lanka
}

\author{
Thilina Abeygunasekara ${ }^{1}$, Sarath Lekamwasam ${ }^{2}$, Janaka Lenora ${ }^{3}$, Gayani Alwis ${ }^{4}$ \\ ${ }^{1}$ Department of Nursing, Faculty of Allied Health Sciences, University of Ruhuna, Matara, Sri Lanka \\ ${ }^{2}$ Department of Medicine, Faculty of Medicine, University of Ruhuna, Matara, Sri Lanka \\ ${ }^{3}$ Department of Physiology, Faculty of Medicine, University of Ruhuna, Matara, Sri Lanka \\ ${ }^{4}$ Department of Anatomy, Faculty of Medicine, University of Ruhuna, Matara, Sri Lanka
}

Corresponding Author:

Thilina Abeygunasekara, BSc

Department of Nursing, Faculty of

Allied Health Sciences, University of

Ruhuna, Matara, Sri Lanka.

E-mail: thilinauwa@ahs.ruh.ac.lk

ORCID:

https://orcid.org/0000-0001-6653-525X

Received: February 1, 2021

Revised: June 2, 2021

Accepted: June 2, 2021
Background: Data on the functional outcomes of hip fracture patients in Sri Lanka are limited. As this information is required for the design of long-term care plans, we assessed the physical activities (activities of daily living $[A D L]$ ) and quality of life $(\mathrm{O} o L)$ of hip fracture survivors in Sri Lanka. Methods: A group of 180 consecutive patients with incident hip fractures admitted to a tertiary care center in Southern Sri Lanka were followed up for 12 months. The Sinhala versions of the Barthel Index, 36-Item Short-Form Survey, and Mini-Mental State Examination were used to assess ADL, QoL, and mental status, respectively. Results: Of the 180 patients (149 women), 107 underwent surgery. An initial sharp decline and partial recovery of $A D L$ and $\mathrm{QoL}$ were observed among patients with hip fractures. Furthermore, patients who underwent surgical treatment showed faster recovery of ADL and $\mathrm{QoL}$ than did patients who were managed conservatively. Similarly, patients who did not have complications during the hospital stay showed faster recovery of $\mathrm{ADL}$ and $\mathrm{QoL}$ than did patients with one or more complications. Conclusions: Hip fractures profoundly affected both $A D L$ and $Q o L$, and recovery remained incomplete at 12 months post fracture. Patients who underwent surgery had a faster recovery than did patients who did not undergo surgery; similarly, patients without complications also had a faster recovery than did those with complications.

Key Words: Quality of life, Sri Lanka, Functional status, Hip fractures

\section{INTRODUCTION}

Hip fracture is considered the most ominous clinical outcome of osteoporosis because of the high mortality, morbidity, and healthcare costs. ${ }^{1,2)}$ Patients with hip fractures have higher mortality rates during their hospital stays and for years after discharge. ${ }^{2)}$ Following a hip fracture, mortality is higher during the initial 6 months than the next 6 months, and compared to that in an age-matched normal population, this increased mortality persists for several years. ${ }^{3,4)}$ Johnston et al..$^{5)}$ observed that mortality among patients aged $>85$ years with hip fractures tended to return to the level in the background population after $2-5$ years, whereas increased mortality persisted beyond 8 years post fracture among those $<85$ years. Apart from mortality related to hip fractures, deaths due to comorbidities also account for the increased mortality apparent after such fractures. ${ }^{6}$

In addition to excess mortality, hip fractures are also associated with high healthcare costs, which vary among countries. A Canadian study in 2013 estimated a mean attributable cost in the first year after fracture of Can\$36,929 among women and Can\$39,479 among men, translating to Can $\$ 282$ million in directly attributable healthcare costs in Ontario and Can $\$ 1.1$ billion in Canada annually. ${ }^{7)}$ Further, a similar study in New Zealand ${ }^{8)}$ reported a combined total cost over 2 years post operation for hip fracture of 
NZ\$66,637,355. According to Cheung et al.,9) the direct cost of hip fractures will increase from US $\$ 9.5$ billion in 2018 to US $\$ 15$ billion in 2050 in nine member countries of the Asian Federation of Osteoporosis Societies.

Aside from the economic implications, impairment of physical function and quality of life (QoL) frequently occurs among hip fracture survivors. Nearly $40 \%-50 \%$ of hip fracture survivors have limitations in at least one activity of daily living (ADL) 12 months after a fracture. ${ }^{10,11)}$ Studies have also shown a sharp reduction in physical function and QoL immediately after hip fracture, with partial recovery later. ${ }^{12-14)}$ The high physical dependency in the post-fracture period is an added burden on caregivers and family members, leading to the institutionalization of patients who are severely affected. $\mathrm{Lin}$ and $\mathrm{Lu}^{15)}$ observed that $56.7 \%$ of caregivers in Taiwan reported "feeling exhausted" because of the added workload of caring for family members with hip fractures.

Asia, with the expanding elderly population, will become the epicenter of hip fractures. On the basis of epidemiological data from four Asian countries-Singapore, Malaysia, Thailand, and Hong Kong-Lau et al. ${ }^{16)}$ predicted that hip fractures would become a major public health challenge in this region. Cheung et al. ${ }^{9}$ predicted that the number of hip fractures would increase from 1,124,060 in 2018 to 2,563,488 in 2050 in the Asian region. Despite these alarming predictions, the preparedness of these countries to meet the challenges of hip fractures is not clearly evident. In South Asian countries, epidemiological data on patients with hip fracture related to causation and short- and long-term clinical outcomes are sparse compared to those in Western countries. This information gap is a major limitation to resource allocation and the design of health and social care pathways. More studies are needed to inform the relevant authorities to make changes in the current health and social care services to face the predicted burden of hip fractures in these countries.

This 12-month follow-up study assessed the physical dependence and QoL of patients admitted with incident hip fractures to a tertiary care center in Southern Sri Lanka. After an extensive search of the major electronic databases, we were unable to identify previous studies examining the QoL of hip fracture survivors in Sri Lanka.

\section{MATERIALS AND METHODS}

This prospective cohort study did not include a parallel control group. Follow-up data from a hip fracture registry maintained since September 2017 were used for this analysis. One hundred eighty patients with incident fragility hip fractures (FHFs) admitted consecutively to a tertiary care center (Teaching Hospital, Karapitiya,
Galle) in the southern province of Sri Lanka were followed up for 12 months. Written informed consent was obtained from all patients or their immediate family members before recruitment. This study included only those patients with new hip fractures resulting from a simple fall (from a standing height or less) and excluded patients with high-energy (non-fragility) fractures and readmissions for the same fracture. The study was approved by the Ethics Review Committee of the Faculty of Medicine, University of Ruhuna, Sri Lanka (No. 19.12.2016: 3.3).

In this study, we applied the validated Sinhala version of the 36Item Short-Form Survey $(\mathrm{SF} 36)^{17)}$ to assess the QoL at discharge and at 3, 6, and 12 months post-fracture. We assessed ADL using the Sinhala version of the Barthel Index $(\mathrm{BI})^{18)}$ at five time points: 1 week before the fracture; at the time of discharge; and at 3, 6, and 12 months after the fracture. Finally, we assessed the mental state of the patients at discharge using the validated Sinhala version of the Mini-Mental State Examination (MMSE) ${ }^{19}{ }^{1}$ Members of the research team visited the relevant wards regularly to collect data, and patients were followed up with regular telephone calls and in-person meetings during subsequent hospital visits. Some patients were interviewed at their residences to collect data.

On the basis of the information on comorbidities gathered from patients during the interview and collected from case notes, we calculated the age-adjusted Charlson Comorbidity Index (ACCI) by weighting comorbid conditions and adding additional points for each decade over 40 years of age. ${ }^{20,21)}$

Data are presented as mean \pm standard deviation, median (interquartile range), or number (percentage). We applied the chisquare and independent $\mathrm{t}$-test for categorical and numerical data, respectively, and used analysis of variance (ANOVA) to compare two groups. Statistical significance was set at $\mathrm{p}<0.05$.

\section{RESULTS}

\section{Sociodemographic and Clinical Characteristics of Patients with an FHF}

The study cohort included 180 consecutive patients (149 women) with incident hip fractures admitted during the study period to the Teaching Hospital, Karapitiya, Galle, Sri Lanka. All fractures occurred following minor falls from a standing height or less. Table 1 presents the sociodemographic and clinical characteristics of the patients with FHF according to the management of their hip fractures. Seven patients (59\%) underwent surgery; the others were managed conservatively (non-surgically). Five patients died while in the hospital, whereas 28 died during the 12-month follow-up. Furthermore, 53 patients (29\%) developed one or more complications during their hospital stay. These complications included 
pressure ulcers (14 patients, $7.8 \%$ ), respiratory tract infections (10 patients, 5.6\%), urosepsis (19 patients, 10.6\%), and acute myocardial ischemia (4 patients, $2.2 \%$ ) (Table 2).

Table 1. Sociodemographic and clinical characteristics of patients with surgically and conservatively managed fragility hip fractures

\begin{tabular}{lcc}
\hline & \multicolumn{2}{c}{ FHF patient management $(\mathrm{n}=180)$} \\
\cline { 2 - 3 } Characteristic & $\begin{array}{c}\text { Surgical } \\
(\mathrm{n}=107)\end{array}$ & $\begin{array}{c}\text { Conservative } \\
(\mathrm{n}=73)\end{array}$ \\
\hline Sex, female & $90(84.1)$ & $59(80.8)$ \\
Age $(\mathrm{y})$ & $73.6 \pm 8.0$ & $82.0 \pm 8.5$ \\
History of previous fragility fracture & $8(7.5)$ & $6(8.2)$ \\
Family history of fragility fracture & $9(8.4)$ & $7(9.6)$ \\
Smoking & $7(6.5)$ & $7(9.6)$ \\
Alcohol consumption & $9(8.4)$ & $10(13.7)$ \\
Use of glucocorticoids & $32(29.9)$ & $19(26.0)$ \\
Comorbid conditions & & \\
Vascular diseases & $39(36.4)$ & $32(43.8)$ \\
Dementia & $2(1.9)$ & $10(13.7)$ \\
COPD/bronchial asthma & $23(21.5)$ & $22(30.1)$ \\
Rheumatic diseases & $3(2.8)$ & $3(4.1)$ \\
Peptic ulcer disease & $14(13.1)$ & $4(5.5)$ \\
Liver disease & $5(4.7)$ & $5(6.8)$ \\
Diabetes mellitus & $15(14.0)$ & $21(28.8)$ \\
Renal diseases & $0(0)$ & $4(5.5)$ \\
Hypertension & $34(31.8)$ & $35(47.9)$ \\
Hyperlipidemia & $10(9.3)$ & $3(4.1)$ \\
\hline
\end{tabular}

Values are presented as number of patients (\%) or mean \pm standard deviation. COPD, chronic obstructive pulmonary disease.

\section{Physical Dependence and QoL of Patients with Hip Fractures during 12 Months Post-fracture}

An initial sharp decline and partial recovery in $\mathrm{ADL}$ were observed among patients with hip fractures (Table 3). A similar pattern was evident in the total score and physical and psychological domains of the SF-36 (Table 4). In addition, the ADL score at 12 months was below the pre-fracture score.

\section{Determinants of QoL at 12 Months}

In the correlation analysis, age and ACCI were found to be inversely related to the two domains of QoL, whereas BI measured at five time points showed positive correlations (Table 5).

Analysis of the trends of BI and SF-36 values over time showed a steady increase in all indices in the group of patients who underwent surgery. In contrast, no major improvement in QoL indices was observed, as ADL decreased slightly over time (Table 6) in pa-

Table 3. Changes in the physical dependence of patients with hip fractures during 12 months post-fracture

\begin{tabular}{lcc}
\hline Time of assessment & Barthel index & Physically dependent subjects \\
\hline Before fracture $(\mathrm{n}=180)$ & $100(95-100)$ & $10(5.6)$ \\
At discharge $(\mathrm{n}=175)$ & $30(20-45)$ & $175(100)$ \\
At 3 months $(\mathrm{n}=172)$ & $40(15-60)$ & $172(100)$ \\
At 6 months $(\mathrm{n}=159)$ & $55(20-80)$ & $152(95.6)$ \\
At 12 months $(\mathrm{n}=147)$ & $85(30-90)$ & $87(59.2)$ \\
\hline
\end{tabular}

Values are presented as median (interquartile range) or number of patients (\%).

${ }^{\text {a) }}$ Barthel index $<90$.

Table 2. Comparisons of complications between surgically and conservatively managed patients with fragility hip fractures

\begin{tabular}{|c|c|c|c|}
\hline \multirow{2}{*}{ Complications } & \multicolumn{2}{|c|}{ FHF patient management $(\mathrm{n}=180)$} & \multirow{2}{*}{ p-value ${ }^{a}$} \\
\hline & Surgical $(\mathrm{n}=107)$ & Conservative $(n=73)$ & \\
\hline Pressure ulcer & $3(2.8)$ & $11(15.1)$ & 0.003 \\
\hline Wound infection & $1(0.9)$ & $8(11.0)$ & 0.002 \\
\hline Urinary tract infection & $7(6.5)$ & $12(16.4)$ & 0.034 \\
\hline Acute renal failure & $1(0.9)$ & $2(2.7)$ & 0.353 \\
\hline Cardiac complication & $1(0.9)$ & $4(5.5)$ & 0.068 \\
\hline Pneumonia & $2(1.9)$ & $8(11.0)$ & 0.009 \\
\hline Myocardial infarction & $2(1.9)$ & $2(2.7)$ & 0.697 \\
\hline Cerebrovascular accident & $2(1.9)$ & $3(4.1)$ & 0.369 \\
\hline Pulmonary embolism & $0(0)$ & $3(4.1)$ & 0.034 \\
\hline Deep venous thrombosis & $0(0)$ & $3(4.1)$ & 0.034 \\
\hline Gastrointestinal bleeding & $3(2.8)$ & $0(0)$ & 0.149 \\
\hline \multicolumn{4}{|l|}{ Number of complication(s) } \\
\hline 0 & $91(85.0)$ & $36(49.3)$ & $<0.001$ \\
\hline 1 & $12(11.2)$ & $21(28.8)$ & $<0.001$ \\
\hline $2+$ & $4(3.7)$ & $16(21.9)$ & $<0.001$ \\
\hline
\end{tabular}

Values are presented as number of patients (\%).

${ }^{\text {a) }}$ Chi-square test. 
Table 4. Changes in SF-36 total scores and physical and psychological domains

\begin{tabular}{lcccc}
\hline Measure & At discharge $(\mathrm{n}=175)$ & At 3 months $(\mathrm{n}=172)$ & At 6 months $(\mathrm{n}=159)$ & At 12 months $(\mathrm{n}=147)$ \\
\hline Physical health & $9.6(6.7-11.7)$ & $19.3(10.2-22.7)$ & $33.8(13.1-45.6)$ & $44.6(16.5-56.7)$ \\
Psychological health & $13.3(11.7-15.0)$ & $28.3(15.4-31.7)$ & $44.6(15.4-58.8)$ & $60.8(14.2-73.0)$ \\
Total SF-36 score & $11.0(7.9-12.8)$ & $22.0(11.1-25.7)$ & $34.0(10.7-49.9)$ & $40.0(8.1-60.4)$ \\
\hline
\end{tabular}

Values are presented as median (interquartile range).

SF-36, 36-item Short-Form Health Survey questionnaire.

Table 5. Correlations of two domains of the SF-36 at 12 months with selected variables

\begin{tabular}{lccc}
\hline \multirow{2}{*}{ Variable } & \multicolumn{2}{c}{ SF-36 at 12 months } & \multirow{2}{*}{ p-value $^{\mathrm{a})}$} \\
\cline { 2 - 3 } Age & Physical health domain & Psychological health domain & $<0.001$ \\
ACCI & -0.53 & -0.48 & $<0.001$ \\
BI before fracture & -0.42 & -0.38 & $<0.001$ \\
BI at discharge & 0.33 & 0.35 & $<0.001$ \\
BI at 3 months & 0.61 & 0.59 & $<0.001$ \\
BI at 6 months & 0.86 & 0.85 & $<0.001$ \\
BI at 12 months & 0.89 & 0.89 & $<0.001$ \\
MMSE at discharge & 0.90 & 0.90 & $<0.001$ \\
\hline
\end{tabular}

SF-36, 36-item Short-Form Health Survey questionnaire; ACCI, age-adjusted Charlson Comorbidity Index; BI, Barthel Index; MMSE, Mini-Mental State Examination.

${ }^{\text {a) }}$ Pearson correlation.

Table 6. Trends in BI and SF-36 scores during follow-up according to the type of treatment and the presence or absence of complications

\begin{tabular}{|c|c|c|c|c|c|c|}
\hline & \multirow{2}{*}{ At discharge } & \multirow{2}{*}{ At 3 months } & \multirow{2}{*}{ At 6 months } & \multirow{2}{*}{ At 12 months } & \multicolumn{2}{|c|}{ p-value $e^{a}$} \\
\hline & & & & & Within-group & Intergroup \\
\hline Surgical & $(n=106)$ & $(\mathrm{n}=105)$ & $(\mathrm{n}=103)$ & $(\mathrm{n}=101)$ & & \\
\hline SF-36 physical & $11.3 \pm 2.9$ & $24.7 \pm 8.8$ & $41.6 \pm 9.9$ & $51.7 \pm 10.8$ & $<0.001$ & $<0.001$ \\
\hline SF-36 psychological & $14.1 \pm 2.5$ & $33.8 \pm 9.9$ & $54.9 \pm 14.0$ & $66.8 \pm 14.4$ & $<0.001$ & $<0.001$ \\
\hline Conservative & $(\mathrm{n}=69)$ & $(n=67)$ & $(\mathrm{n}=56)$ & $(n=46)$ & & \\
\hline SF-36 physical & $5.9 \pm 2.5$ & $9.8 \pm 4.0$ & $11.1 \pm 6.6$ & $11.5 \pm 6.2$ & $<0.001$ & \\
\hline SF-36 psychological & $10.8 \pm 3.4$ & $12.7 \pm 3.9$ & $12.2 \pm 5.7$ & $12.5 \pm 7.8$ & $<0.001$ & \\
\hline \multicolumn{7}{|l|}{ BI } \\
\hline Surgical BI & $37.7 \pm 10.4$ & $56.0 \pm 14.7$ & $68.7 \pm 15.5$ & $85.8 \pm 12.0$ & $<0.001$ & $<0.001$ \\
\hline Conservative BI & $24.1 \pm 9.7$ & $17.0 \pm 7.5$ & $16.3 \pm 7.6$ & $16.6 \pm 10.0$ & $<0.001$ & \\
\hline No complications & $(\mathrm{n}=122)$ & $(\mathrm{n}=121)$ & $(\mathrm{n}=117)$ & $(\mathrm{n}=116)$ & & \\
\hline SF-36 physical & $9.9 \pm 3.8$ & $21.0 \pm 10.6$ & $34.5 \pm 15.7$ & $42.4 \pm 20.3$ & $<0.001$ & $>0.001$ \\
\hline SF-36 psychological & $13.3 \pm 2.9$ & $28.7 \pm 12.9$ & $44.5 \pm 22.6$ & $54.1 \pm 26.6$ & $<0.001$ & $>0.001$ \\
\hline $\mathrm{BI}$ & $33.3 \pm 13.1$ & $45.2 \pm 23.6$ & $53.1 \pm 30.0$ & $63.8 \pm 37.2$ & $<0.001$ & $<0.001$ \\
\hline With complications & $(\mathrm{n}=53)$ & $(\mathrm{n}=51)$ & $(n=42)$ & $(\mathrm{n}=31)$ & & \\
\hline SF-36 physical & $7.5 \pm 3.4$ & $13.7 \pm 7.4$ & $20.6 \pm 16.5$ & $26.5 \pm 19.0$ & $<0.001$ & \\
\hline SF-36 psychological & $11.6 \pm 3.6$ & $18.4 \pm 10.7$ & $26.7 \pm 23.0$ & $33.6 \pm 28.7$ & $<0.001$ & \\
\hline $\mathrm{BI}$ & $26.5 \pm 11.7$ & $24.2 \pm 16.8$ & $23.7 \pm 22.5$ & $25.0 \pm 31.0$ & $<0.001$ & \\
\hline
\end{tabular}

Values are presented as mean \pm standard deviation.

BI, Barthel Index; SF-36, 36-item Short-Form Health Survey questionnaire.

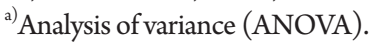

tients who were managed conservatively. We observed similar trends for the groups of patients with one or more or no complications during their hospital stay (Table 6).

\section{DISCUSSION}

This analysis revealed a sharp decline in ADL and QoL after a hip 
fracture and a gradual but partial recovery over the next 12 months. The ADL score of patients with FHF at 12 months did not reach the pre-fracture level, and nearly $60 \%$ still had a limitation of at least one $\mathrm{ADL}$ function at 12 months post fracture.

We observed that age and comorbidity negatively impacted QoL at 12 months post hip fracture, whereas ADL, both pre-and post-fracture $\mathrm{ADL}$, and MMSE score at discharge showed positive influences on QoL. Patients managed surgically showed steady improvements in both ADL and QoL over the next 12 months compared with patients who were managed non-surgically. Similarly, patients without complications during hospital stay showed greater recovery of $\mathrm{ADL}$ and $\mathrm{QoL}$ than did patients with complications.

Physical dependence and poor QoL after hip fracture are common observations, and the findings of the current study are consistent with those reported previously. Analyzing a large cohort of 10,325 patients, Gjertsen et al. ${ }^{22)}$ noticed a marked reduction in QoL, and 58\% of patients reported walking difficulty at 12 months post fracture. Hall et al. ${ }^{10)}$ also observed reduced QoL, difficulties in maintaining balance, and reduced physical activity in patients with hip fracture compared to those in age-matched controls. Furthermore, patients with hip fractures were unable to achieve pre-fracture mobility and independence. Many other studies have also reported the negative impact of hip fractures on QoL and physical function, including those by Milte et al.," ${ }^{23)}$ Prieto-Alhambra et al., ${ }^{12)}$ Tarride et al., ${ }^{24)}$ Lim, $^{25)}$ and Amarilla-Donoso et al. ${ }^{13)}$

In this study, age and comorbidity negatively affected the $\mathrm{QoL}$ of patients with hip fractures, whereas cognition and function (represented by MMSE) and independence in ADL positively affected the QoL of patients with hip fractures. Furthermore, patients treated surgically and those without complications had better outcomes. Amarilla-Donoso et al. ${ }^{13)}$ also reported that health-related QoL (HRQoL) scores at 1-month post-fracture was related to pre-fracture $\mathrm{BI}$, depression, and surgery type. The influence of pre-fracture BI and MMSE on the QoL of hip fracture survivors has also been reported by others. ${ }^{26)}$ While Gjertsen et al. ${ }^{22)}$ and Milte et $\mathrm{al}^{23)}$ showed the influence of age on the outcome of patients with hip fractures, Tarride et al. ${ }^{24)}$ found that, among many predictors, mobility was a strong predictor of QoL in these patients. Moreover, although they observed a partial recovery of HRQoL after 1 month, it remained below the pre-fracture level even 36 months post-fracture.

The positive association between $\mathrm{ADL}$ and QoL suggests the need for caregivers to focus on interventions that enhance the physical independence of patients with hip fractures. Unlike age and comorbidities, $\mathrm{ADL}$ is a modifiable determinant of QoL. Thus, attempts must be made to improve the QoL of these patients by improving their ADL. However, this concept needs to be tested in an interventional study before implementation.

Our observations reconfirm the debilitating nature of hip fractures. Although a gradual improvement in both $\mathrm{ADL}$ and $\mathrm{QoL}$ is observed during the first year, patients did not reach pre-fracture ADL, and their QoL remained lower. Furthermore, nearly 58\% of patients were not fully physically active at 12 -month post-fracture. Poor QoL and $\mathrm{ADL}$ make patients with hip fractures a burden for their families and caregivers. ${ }^{15)}$

Only $59 \%$ of patients in our study group had undergone surgery. The reasons for non-surgical management included advanced age, higher comorbidity, and lack of consent for the procedure. The $\mathrm{ADL}$ and $\mathrm{QoL}$ at each follow-up were greater among patients who had undergone surgery than among those who were managed conservatively. Compared with non-surgical management, surgery, particularly when performed early, showed advantages related to mortality, ADL, and QoL in hip fracture patients. ${ }^{27)}$ Yoon et al. ${ }^{28)}$ reported higher cumulative mortality rates for up to 2 years and a higher prevalence of non-functional ambulatory state among hip fracture patients managed conservatively than among those who had undergone surgery. Furthermore, higher rates of complications ( $12.5 \%$ vs. $6.6 \%)$ and mortality (7.4\% vs. $1.7 \%)$ were observed among hip fracture patients managed conservatively than among those managed surgically. ${ }^{29)}$

In the current study, all patients were followed up for 12 months post-fracture, except for 33 patients who died during the 12-month follow-up. This can be considered a strength of this study. The study, however, was limited to one area of the country; thus, studies of similar nature from other parts of the country are needed before generalization of the results. Another limitation was that we did not assess other measurements of physical function such as gait speed or chair rise in this study.

In conclusion, hip fractures led to a rapid decline in ADL and QoL immediately after the event, with a gradual but partial recovery during the first 12 months post-fracture. We observed significant differences in the $\mathrm{ADL}$ and $\mathrm{QoL}$ between surgically and conservatively treated patients and between those who did and did not have complications. On the basis of these findings, we urge relevant authorities to take steps to increase the rate of surgical treatment for patients with hip fractures. To achieve this, the barriers and limitations of surgical treatment should be assessed and the necessary steps be taken to address them. Furthermore, steps should also be taken to avoid post-fracture complications. Most complications can be avoided by adhering to proper nursing practices, venous thromboembolism prophylaxis, and early mobilization. 


\section{ACKNOWLEDGMENTS}

\section{CONFLICT OF INTEREST}

The researchers claim no conflicts of interest.

\section{FUNDING}

This study was supported by the Faculty Research Grant in 2017 from Faculty of Medicine, University of Ruhuna, Matara, Sri Lanka, and the University Grants Commission, Colombo, Sri Lanka (No. UGC/VC/DRIC/PG2017(1)/RUH/01).

\section{AUTHOR CONTRIBUTIONS}

Conceptualization, TA, SL, JL, GA; Data curation, TA; Formal analysis, TA, SL; Funding acquisition, TA, SL; Investigation, TA; Methodology, TA, SL, JL, GA; Project administration, TA, SL, JL, GA; Supervision, SL, JL, GA; Writing-original draft, TA, SL; Writing-review \& editing, TA, SL.

\section{REFERENCES}

1. Abrahamsen B, van Staa T, Ariely R, Olson M, Cooper C. Excess mortality following hip fracture: a systematic epidemiological review. Osteoporos Int 2009;20:1633-50.

2. Braithwaite RS, Col NF, Wong JB. Estimating hip fracture morbidity, mortality and costs. J Am Geriatr Soc 2003;51:364-70.

3. Brozek W, Reichardt B, Kimberger O, Zwerina J, Dimai HP, Kritsch D, et al. Mortality after hip fracture in Austria 2008-2011. Calcif Tissue Int 2014;95:257-66.

4. Brossa Torruella A, Tobias Ferrer J, Zorrilla Ribeiro J, Lopez Borras E, Alabart Teixido A, Belmonte Garridof M. Mortalidad a los tres anos de los pacientes con fractura de femur [Mortality after hip fracture: a three year follow-up study]. Med Clin (Barc) 2005; 124:53-4

5. Johnston AT, Barnsdale L, Smith R, Duncan K, Hutchison JD. Change in long-term mortality associated with fractures of the hip: evidence from the Scottish hip fracture audit. J Bone Joint Surg Br 2010;92:989-93.

6. Kanis JA, Oden A, Johnell O, De Laet C, Jonsson B, Oglesby AK. The components of excess mortality after hip fracture. Bone 2003;32:468-73.

7. Nikitovic M, Wodchis WP, Krahn MD, Cadarette SM. Direct health-care costs attributed to hip fractures among seniors: a matched cohort study. Osteoporos Int 2013;24:659-69.

8. Lane A. Direct costs of osteoporosis for New Zealand women. Pharmacoeconomics 1996;9:231-45.

9. Cheung CL, Ang SB, Chadha M, Chow ES, Chung YS, Hew FL, et al. An updated hip fracture projection in Asia: the Asian Fed- eration of Osteoporosis Societies study. Osteoporos Sarcopenia 2018;4:16-21.

10. Hall SE, Williams JA, Senior JA, Goldswain PR, Criddle RA. Hip fracture outcomes: quality of life and functional status in older adults living in the community. Aust N Z J Med 2000; 30:327-32.

11. Lekamwasam S, Sabapathippillai S. Mortality and physical dependence following fragility hip fracture: data from a regional hip fracture registry in Sri Lanka. Arch Osteoporos 2019;14:97.

12. Prieto-Alhambra D, Moral-Cuesta D, Palmer A, Aguado-Maestro I, Bardaji MF, Branas F, et al. The impact of hip fracture on health-related quality of life and activities of daily living: the SPARE-HIP prospective cohort study. Arch Osteoporos 2019; 14:56.

13. Amarilla-Donoso FJ, Lopez-Espuela F, Roncero-Martin R, Leal-Hernandez O, Puerto-Parejo LM, Aliaga-Vera I, et al. Quality of life in elderly people after a hip fracture: a prospective study. Health Qual Life Outcomes 2020;18:71.

14. Kang JH, Lee G, Kim KE, Lee YK, Lim JY. Determinants of functional outcomes using clinical pathways for rehabilitation after hip fracture surgery. Ann Geriatr Med Res 2018;22:26-32.

15. Lin PC, Lu CM. Hip fracture: family caregivers' burden and related factors for older people in Taiwan. J Clin Nurs 2005; 14:71926.

16. Lau EM, Lee JK, Suriwongpaisal P, Saw SM, Das De S, Khir A, et al. The incidence of hip fracture in four Asian countries: the Asian Osteoporosis Study (AOS). Osteoporos Int 2001;12:23943.

17. Gunawardena NS, Seneviratne SR, Atauda T. An approach to validation of a multi-dimensional tool.J Coll Community Physicians Sri Lanka 2003;8:18-26.

18. Lekamwasam S, Karunatilake K, Kankanamge SK, Lekamwasam V. Physical dependency of elderly and physically disabled; measurement concordance between 10-item Barthel index and 5-item shorter version. Ceylon Med J 2011;56:114-8.

19. de Silva HA, Gunatilake SB. Mini Mental State Examination in Sinhalese: a sensitive test to screen for dementia in Sri Lanka. Int J Geriatr Psychiatry 2002;17:134-9.

20. Charlson M, Szatrowski TP, Peterson J, Gold J. Validation of a combined comorbidity index. J Clin Epidemiol 1994;47:124551.

21. Charlson ME, Pompei P, Ales KL, MacKenzie CR. A new method of classifying prognostic comorbidity in longitudinal studies: development and validation. J Chronic Dis 1987;40:373-83.

22. Gjertsen JE, Baste V, Fevang JM, Furnes O, Engesæter LB. Quality of life following hip fractures: results from the Norwegian hip fracture register. BMC Musculoskelet Disord 2016;17:265. 
23. Milte R, Crotty M, Miller MD, Whitehead C, Ratcliffe J. Quality of life in older adults following a hip fracture: an empirical comparison of the ICECAP-O and the EQ-5D-3 L instruments. Health Qual Life Outcomes 2018;16:173.

24. Tarride JE, Burke N, Leslie WD, Morin SN, Adachi JD, Papaioannou A, et al. Loss of health related quality of life following low-trauma fractures in the elderly. BMC Geriatr 2016;16:84.

25. Lim JY. Fragility fracture care: an urgent need to implement the integrated model of geriatric care. Ann Geriatr Med Res 2019; 23:1-2.

26. Buecking B, Struewer J, Waldermann A, Horstmann K, Schubert $\mathrm{N}$, Balzer-Geldsetzer $\mathrm{M}$, et al. What determines health-related quality of life in hip fracture patients at the end of acute care? A prospective observational study. Osteoporos Int 2014;25:475-
84.

27. Bohm E, Loucks L, Wittmeier K, Lix LM, Oppenheimer L. Reduced time to surgery improves mortality and length of stay following hip fracture: results from an intervention study in a Canadian health authority. Can J Surg 2015;58:257-63.

28. Yoon BH, Baek JH, Kim MK, Lee YK, Ha YC, Koo KH. Poor prognosis in elderly patients who refused surgery because of economic burden and medical problem after hip fracture. J Korean Med Sci 2013;28:1378-81.

29. Tan ST, Tan WP, Jaipaul J, Chan SP, Sathappan SS. Clinical outcomes and hospital length of stay in 2,756 elderly patients with hip fractures: a comparison of surgical and non-surgical management. Singapore Med J 2017;58:253-7. 